\title{
Multicenter study of zoledronic acid administration in non-small-cell lung cancer patients with bone metastasis: Thoracic Oncology Research Group (TORG) 1017
}

\author{
YOSHIRO NAKAHARA $^{1-3}$, YUKIO HOSOMI ${ }^{1}$, MASAHIKO SHIBUYA ${ }^{1}$, HISASHI MITSUFUJI ${ }^{4}$, \\ MASATO KATAGIRI $^{5}$, KATSUHIKO NAOKI ${ }^{2,6,7}$, KENZO SOEJIMA ${ }^{6,7}$, NAOYUKI NOGAMI $^{8}$, \\ SEISUKE NAGASE ${ }^{9,10}$, MASANORI NISHIKAWA ${ }^{11}$, KOICHI MINATO $^{12}$, YUICHI TAKIGUCHI $^{13}$, \\ NOBUHIKO SEKI ${ }^{14}$, KOUZO YAMADA ${ }^{3}$, TAKASHI SETO ${ }^{15}$ and HIROAKI OKAMOTO ${ }^{16}$
}

\begin{abstract}
${ }^{1}$ Department of Thoracic Oncology and Respiratory Medicine, Tokyo Metropolitan Cancer and Infectious Disease Center Komagome Hospital, Tokyo 113-0021; ${ }^{2}$ Department of Respiratory Medicine, Kitasato University School of Medicine, Kanagawa 252-0374; ${ }^{3}$ Department of Thoracic Oncology, Kanagawa Cancer Center, Kanagawa 241-8515; ${ }^{4}$ Kitasato University

School of Nursing, Kanagawa 252-0329; ${ }^{5}$ School of Allied Health Sciences, Kitasato University, Kanagawa 252-0373;

${ }^{6}$ Division of Pulmonary Medicine, Department of Medicine; ${ }^{7}$ Cancer Center, Keio University School of Medicine, Tokyo

160-8582; ${ }^{8}$ Department of Thoracic Oncology and Medicine, National Hospital Organization Shikoku Cancer Center, Ehime 791-0280; ${ }^{9}$ Respiratory Disease Center, International University of Health and Welfare, Mita Hospital, Tokyo 108-8329;

${ }^{10}$ Department of Thoracic Surgery, Tokyo Medical University, Tokyo 160-0023; ${ }^{11}$ Department of Respiratory Medicine,

Fujisawa City Hospital, Kanagawa 251-8550; ${ }^{12}$ Department of Respiratory Medicine, Gunma Prefectural Cancer Center, Gunma 373-8550; ${ }^{13}$ Department of Medical Oncology, Graduate School of Medicine, Chiba University, Chiba 260-8670;

${ }^{14}$ Division of Medical Oncology, Department of Internal Medicine, Teikyo University School of Medicine, Tokyo 173-8606;

${ }^{15}$ Department of Thoracic Oncology, National Hospital Organization Kyushu Cancer Center, Fukuoka 811-1395;

${ }^{16}$ Department of Respiratory Medicine, Yokohama Municipal Citizen's Hospital, Kanagawa 240-8555, Japan
\end{abstract}

Received September 5, 2018; Accepted June 10, 2019

DOI: $10.3892 /$ mco.2019.1903

\begin{abstract}
Skeletal-related events (SREs) may occur at the time of first diagnosis in $20-30 \%$ of lung cancer patients with bone metastases. Several clinical trials have shown that zoledronic acid (ZA) is effective for decreasing SREs. The main objective of the present study was to discuss clinical data of ZA and compare the frequency of SREs with previous reports. All patients with non-small-cell lung cancer (NSCLC) with metastatic bone disease who were administered ZA at least twice
\end{abstract}

Correspondence to: Dr Yoshiro Nakahara, Department of Respiratory Medicine, Kitasato University School of Medicine, 1-15-1 Kitasato, Minami-ku, Sagamihara, Kanagawa 252-0374, Japan E-mail:md100062@kitasato-u.ac.jp

Abbreviations: BMA, bone-modifying agent; BMAs, bone-modifying agents; IGF, insulin-like growth factor; JSMO, Japanese Society of Medical Oncology; NSCLC, non-small-cell lung cancer; ONJ, osteonecrosis of the jaw; PS, performance status; RANKL, receptor activator of nuclear factor $\kappa \mathrm{B}$ ligand; SRE, skeletal-related event; SREs, skeletal-related events; TGF- $\beta$, transforming growth factor- $\beta$; ZA, zoledronic acid

Key words: zoledronic acid, non-small-cell lung cancer, bone metastases between January 2008 and December 2009 were eligible for inclusion in the study. In total, 198 consecutive patients were identified. The median duration of $\mathrm{ZA}$ administration was 106 days [95\% confidence interval (CI), 92-133 days], and the median number of ZA administrations was 4 (range, 2-41). The median time to first SRE in patients who experienced SRE following ZA treatment was 202 days (95\% CI, 156-264 days). Among the 78 patients who had already experienced SRE prior to ZA treatment, 35 (45\%) experienced SRE subsequently after starting ZA treatment. On the other hand, among the 120 patients without a history of SRE before starting ZA treatment, 42 (35\%) experienced SRE after the start of $\mathrm{ZA}$ administration $(\mathrm{P}=0.16)$. No osteonecrosis of the jaw (ONJ) was reported in any of the patients. The present study revealed that ZA had a certain level of efficacy regardless of the presence or absence of prior SREs. However, the duration of ZA therapy was short in this study; further accumulation of data on the long-term prognosis and incidence rates of $\mathrm{ONJ}$ and other late complications of $\mathrm{ZA}$ therapy seems to be particularly important.

\section{Introduction}

Lung cancer is frequently accompanied by bone metastasis. A prospective observation study conducted in Japan revealed that bone metastasis was present at the time of diagnosis in $48 \%$ of patients with stage IV non-small-cell lung cancer (NSCLC) and 
in $40 \%$ of those with extensive-stage small-cell lung cancer (1). It has also been demonstrated that skeletal-related events (SREs) such as pain, bone fracture, and spinal cord compression in cases of bone metastases lower the patients' quality of life and worsen their prognosis. In cases of lung cancer with bone metastases at an advanced stage, SREs are reportedly found in approximately $20-30 \%$ of cases at the time of diagnosis (1-3). The time between the occurrence of bone metastases and the onset of SREs is reportedly shorter in patients with lung cancer than in those with breast or prostate cancer. Therefore, it is important to initiate treatment as soon as possible after the diagnosis of bone metastases to prevent SREs.

Under normal conditions, osteoclasts resorb bone and release growth factors accumulated in the bone matrix, such as insulin-like growth factor (IGF) and transforming growth factor- $\beta$ (TGF- $\beta$ ), into the marrow, thereby facilitating osteogenesis by osteoblasts. On the other hand, osteoblasts produce the receptor activator of nuclear factor $\kappa \mathrm{B}$ ligand (RANKL), promote the differentiation of osteoclast precursors into osteoclasts, and activate mature osteoclasts. The process of this series of events is called bone remodeling. If cancer cells invade this environment, osteolysis by osteoclasts causes IGF and TGF- $\beta$ to be released from the bone matrix in order to serve as growth factors for cancer cells. Cancer cells release cytokines such as parathyroid hormone-related peptide and prostaglandin E2, which subsequently induce the expression of RANKL. In turn, RANKL activates osteoclasts, and the activated osteoclasts accelerate bone resorption. This secures a space for cancer cells to proliferate and survive and allows IGF and TGF- $\beta$ accumulated in the bone matrix to be released as a means to promote the proliferation of cancer cells. The proliferation of cancer cells increases cytokines that stimulate osteoblasts and further facilitates the activity of osteoclasts, creating a vicious cycle (4).

Bone-modifying agents (BMAs) aim to inhibit the progression of bone metastases and to reduce SREs by severing this vicious cycle of bone metastases. At present, BMAs used for lung cancer include the bisphosphonate zoledronic acid and denosumab, which is a monoclonal antibody for RANKL.

Zoledronic acid accumulates in the surface of the bone matrix and exerts its SRE-inhibiting action by causing apoptosis of osteoclasts that have incorporated zoledronic acid, thereby inhibiting bone resorption $(5,6)$. In a phase III randomized placebo-controlled trial in patients with solid cancers, including non-small cell lung cancer, the incidence rate of SREs (irradiation to bone, pathological fracture, surgical treatment of bone lesions, spinal cord compression, and hypercalcemia) by the 87 th week was significantly decreased by administration of zoledronic acid $(7,8)$.

Based on the results of this study, administration of zoledronic acid in patients with lung cancer accompanied by bone metastases has become the standard treatment in daily clinical practice. The Japanese Society of Medical Oncology (JSMO) guidelines for the diagnosis and treatment of bone metastases also recommend this therapy. However, although osteonecrosis of the jaw (ONJ) is known as an adverse event of zoledronic acid therapy, there have been no comprehensive reports in the field of lung cancer. With this in mind, we conducted the present retrospective multi-center survey study to better understand the clinical course of NSCLC accompanied by bone metastases, paying particular attention to the frequency of ONJ, during a period when zoledronic acid was becoming widely used.

\section{Materials and methods}

Patients. This study covered the observation period between January 2008 and December 2009, and included 12 medical facilities (Table I) that were routinely prescribing zoledronic acid for patients with NSCLC accompanied by bone metastases and that consented to cooperate in the study. All patients with NSCLC with bone metastases who received at least 2 doses of zoledronic acid were enrolled. The protocol was approved by the Clinical Trial Review Committee of the Thoracic Oncology Research Group (TORG) and the Institutional Review Board of TokyoMetropolitan Cancer and Infectious disease Center Komagome Hospital (September 13, 2010; approval no. 863) and by each participating institution. Due to the retrospective nature of the study, the requirement for written informed consent was waived.

The purpose of the study was to evaluate the incidence of ONJ. The study items included age, sex, performance status (PS), histological type, disease stage, treatment regimen, number of zoledronic acid doses, duration of zoledronic acid therapy, types of SREs, time until SRE occurrence, and presence/absence of ONJ.

SREs were defined as bone fracture, spinal cord compression (spinal paralysis), surgery for bone lesions, radiation therapy for bone lesions, and hypercalcemia.

Statistical analysis. Differences in characteristics were evaluated using the $\chi^{2}$ test. Survival was estimated using the Kaplan-Meier method. All the tests were two-sided, and $\mathrm{P}<0.05$ was considered to indicate a statistically significant difference. All the data were analyzed using JMP software, version 10 (SAS Institute, Inc.).

\section{Results}

A total of 198 patients were enrolled from the 12 facilities. Table II shows the patient characteristics. The subjects comprised 126 men and 72 women, with a median age of 64 (44-89) years. The histological type was adenocarcinoma in 131 patients, squamous cell carcinoma in 30, and not otherwise specified in 37 . Seventy-eight patients had experienced SREs prior to the beginning of zoledronic acid therapy, whereas 120 patients had not. Table III shows the regimens of zoledronic acid therapy. The median duration of zoledronic acid therapy was 106 days [95\% confidence interval (CI): 92-133 days] in 196 patients, excluding two in whom data collection was not possible. The median number of zoledronic acid doses was 4 (2-41).

A breakdown of the SREs observed is shown in Fig. 1. Overall, SREs occurred in 77 of 198 patients. The period of survival until the occurrence of the first SRE or death after zoledronic acid therapy (survival without SREs) was 202 (95\% CI: 156-246) days (Fig. 2). In relation to the presence or absence of a history of SREs prior to zoledronic acid therapy, SREs reoccurred during zoledronic acid therapy in 35 of 78 patients $(45 \%)$ with a history of SREs, whereas SREs occurred in 42 of 120 patients $(35 \%)$ without a history of SREs (Fig. 1). There 
Table I. Institutions involved in the study.

Institution name

No. of patients recruited

Tokyo Metropolitan Cancer and Infectious disease Center Komagome Hospital

Keio University School of Medicine

Shikoku Cancer center

Yokohama Municipal Citizen's Hospital

Tokyo Medical University

Kitasato University School of Medicine

Fujisawa city hospital

National Kyushu Cancer Center

Gunma Prefectural Cancer Center

Table II. Patients characteristics.

\begin{tabular}{lc}
\hline Characteristics & No. of patients \\
\hline Total no. of patients & 198 \\
Sex & \\
Male & 126 \\
Female & 72 \\
Age, years & \\
Median & 64 \\
Range & $44-89$ \\
Histology & \\
Adenocarcinoma & 131 \\
Squamous cell carcinoma & 30 \\
NSCLC-NOS & 37 \\
Prior SREs & \\
No & 120 \\
Yes & 78 \\
\hline
\end{tabular}

NSCLC-NOS, non-small-cell lung cancer-not otherwise specified; SREs, skeletal-related events.

was no significant difference in the rate of SRE occurrence between the two groups $(\mathrm{P}=0.16)$. No $\mathrm{ONJ}$ occurred in any of the 198 patients $(95 \% \mathrm{CI}: 0-1.9 \%)$.

\section{Discussion}

This study was a retrospective study in consecutively enrolled patients with advanced NSCLC with bone metastases who received zoledronic acid therapy. The median period of survival until the first SRE or death was 202 days. In a randomized study conducted by Rosen et al (8), the time to the first SRE was 236 days. In our study, the number of events was judged to be insufficient for analysis of the time to the first SRE, and the time to the first SRE, including death as an event, was therefore examined. A subgroup analysis of patients with NSCLC from the aforementioned randomized study showed that the median survival period was 177 days. Our present study showed a longer survival period, but this may have reflected the characteristic features of lung cancer in Japanese patients, who are often positive for driver gene mutations such as EGFR gene mutation. It has also been reported that the risk of SRE occurrence is decreased by $38 \%$ and that the time to the first SRE is significantly longer in patients on zoledronic acid therapy (9).

The-above mentioned subgroup analysis of the randomized controlled study also revealed that the risk of developing SREs was 1.41-fold higher in patients with a history of SREs than in those without such a history (10). In the study, the incidence rates of SREs after zoledronic acid therapy were 42 and 34\% in patients with a history of SREs and in those without such a history. In our study, there was no significant difference in the incidence rate of SREs after the initiation of zoledronic acid therapy between patients who had prior SREs and those who did not. The incidence rates of SREs after zoledronic acid therapy in our study were similar to the-above mentioned analysis (10). In other words, our study showed that zoledronic acid has a certain level of efficacy regardless of the presence or absence of prior SREs.

In addition, Hirsh et al (10) reported that zoledronic acid is slightly more effective in patients with a history of SREs. More specifically, the risk of SREs was decreased by $31 \%$, and the time to the first SRE was increased by 4 months in patients with a history of SREs, whereas the risk of SREs was decreased by $23 \%$ and the time to the first SRE was increased by 2.5 months in those without a history of SREs. We did not examine this issue because no randomization was used in this study (10).

In this study, ONJ was not found in any patient. ONJ associated with the administration of BMAs is defined as follows: The presence of bone fistula occurring from inside or outside the oral cavity or exposed bone persisting for at least 8 weeks in the mouth, jaw, or face area in patients who were on current or prior bone-modifying agent (BMA) therapy and who have no history of radiation therapy or evident metastatic lesions in the jawbone. Although rare, ONJ is a serious adverse event. It has been reported that the incidence rate of $\mathrm{ONJ}$ associated with the use of injectable bisphosphonate for bone metastases depends 
Table III. Duration and number of ZA administrations.

\begin{tabular}{lcc}
\hline Variable & Duration of ZA administration & No. of ZA administrations \\
\hline Total no. of patients & 196 & 197 \\
Median, days or $\mathrm{n}$ & 106 & 4 \\
Range, days & - & $2-41$ \\
$95 \%$ CI & $92-133$ & -
\end{tabular}

ZA, zoledronic acid; CI, confidence interval.

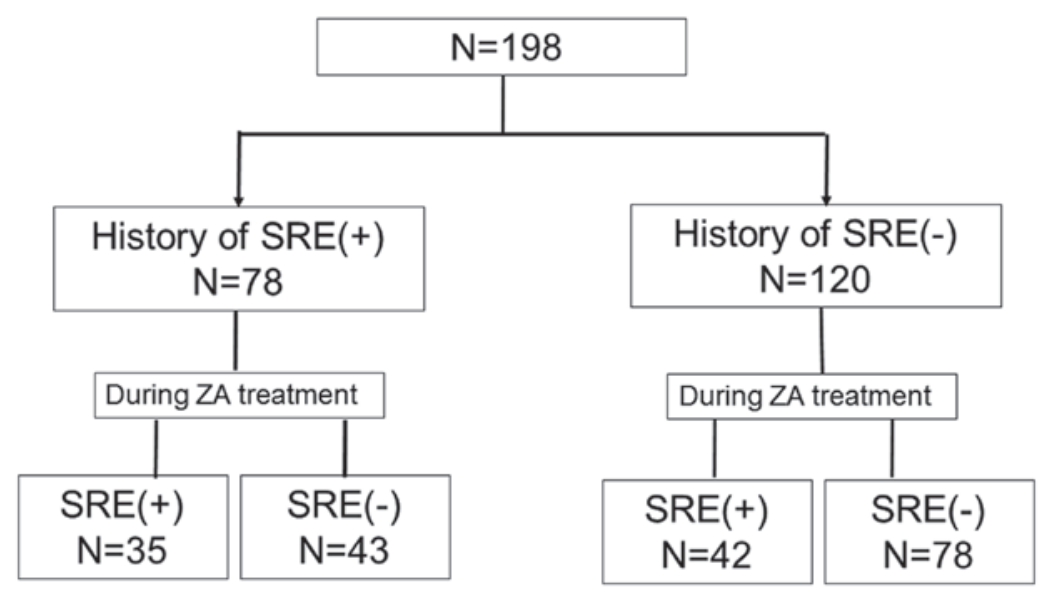

Figure 1. SRE with or without history of SRE prior to ZA administration. Among the 78 patients who had already experienced SRE prior to ZA treatment, 35 (45\%) subsequently experienced SRE following the commencement of ZA treatment. On the other hand, among the 120 patients without a history of SRE prior to starting ZA treatment, 42 (35\%) experienced SRE after the start of ZA administration. SRE, skeletal-related event; ZA, zoledronic acid.

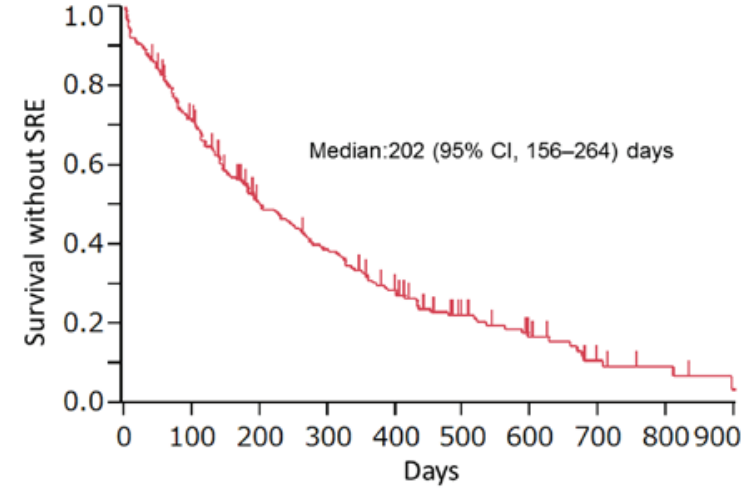

Figure 2. Time to first SRE in patients who experienced SRE following ZA treatment. The median time to the first SRE was 202 days $(95 \% \mathrm{CI}$, 156-264 days) in patients who experienced SRE following ZA treatment. SRE, skeletal-related event; CI, confidence interval; ZA, zoledronic acid.

on the type of bisphosphonate agent, total dose, dosing period, and history of dental disease (11), and the incidence rate is particularly high, at $1.3 \%$, when zoledronic acid, which contains nitrogen, is used (12). Risk factors of ONJ include invasive dental treatment applied to the bone such as tooth extraction, poor status of oral hygiene, periodontal disease, periodontal abscess, and a history of inflammatory disease such as apical periodontitis (13). It has been reported that proper management of oral hygiene reduces the risk of ONJ occurrence during BMA therapy, and it is thus recommended to routinely perform dental checkups and prophylactic dental procedures before the initiation of BMA therapy (14). All facilities that participated in this study were general hospitals that had full-time dentists who provided appropriate oral care at the time of zoledronic acid therapy. The degree of intervention by dentists are various between facilities. However, for example, dentists performed dental checkup for all patients before the administration of zoledronic acid in the Department of Thoracic Oncology and Respiratory Medicine, TokyoMetropolitan Cancer and Infectious disease Center Komagome Hospital. These interventions by expert dentists might have led to the lack of occurrence of ONJ among the subjects in this study. In addition, the longer the duration of the use of injectable bisphosphonate, the higher the risk of ONJ occurrence; the incidence rate of ONJ is reportedly $1.5 \%$ after $4-12$ months of bisphosphonate therapy, whereas the corresponding rate is $7.7 \%$ after $27-48$ months (11). In our study, the median duration of zoledronic acid therapy was only 106 days, and this may be another reason for why there was no occurrence of ONJ.

This study has some limitations. First, this was a retrospective study including a small number of patients. Second, this study did not use a randomized design in relation to the presence or absence of zoledronic acid therapy. Third, the duration of zoledronic acid therapy was short for the clinical situation of Japanese patients, in whom the involvement of driver mutations is frequent. Fourth, this study did not include the data about the treatment for lung cancer and the lesion and radiologic appearance of bone metastases. 
Despite these limitations, we consider that this study is valuable, because the clinical course of NSCLC accompanied by bone metastases and the related occurrence of ONJ have rarely been reported in a comprehensive manner since BMAs have become widely used. In the treatment of NSCLC, many novel drugs, including new molecular-targeted drugs, immune checkpoint inhibitors, and angiogenesis inhibitors, are currently available, achieving marked prolongation of survival. However, the development of new drugs for bone metastases has been limited, and it is expected that zoledronic acid will continue to play an important role in the treatment of bone metastases. In this regard, further accumulation of data on the long-term prognosis and incidence rates of ONJ and other late complications of zoledronic acid therapy seems to be particularly important.

Our study showed that zoledronic acid has a certain level of efficacy regardless of the presence or absence of prior SREs. However, the duration of zoledronic acid therapy was short in this study, further accumulation of data on the long-term prognosis and incidence rates of ONJ and other late complications of zoledronic acid therapy seems to be particularly important.

\section{Acknowledgements}

The authors would like to thank Ms Yumiko Tanabe and Mr. Hiroyuki Kashiro (Thoracic Oncology Research Group, Yokohama, Japan) for assisting with data management during this study.

\section{Funding}

No funding was received.

\section{Availability of data and materials}

The datasets used and/or analyzed during the present study are available from the corresponding author on reasonable request.

\section{Authors' contributions}

YH and MS conceived and designed the study. YN, YH, HM, MK, KN, KS, NN, SN, MN, KM, YT, NS, KY, TS and HO acquired the data. $\mathrm{YN}$ and $\mathrm{YH}$ analyzed the data and wrote the manuscript. All authors gave approval of the final version of the manuscript.

\section{Ethics approval and consent to participate}

The protocol was approved by the Clinical Trial Review Committee of the Thoracic Oncology Research Group (TORG) and the Institutional Review Board of TokyoMetropolitan Cancer and Infectious disease Center Komagome Hospital (September 13, 2010; approval no. 863) and by each participating institution. Due to the retrospective nature of the study, the requirement for written informed consent was waived.

\section{Patient consent for publication}

Not applicable.

\section{Competing interests}

The authors declare that they have no competing interests.

\section{References}

1. Katakami N, Kunikane H, Takeda K, Takayama K, Sawa T, Saito H, Harada M, Yokota S, Ando K, Saito Y, et al: Prospective study on the incidence of bone metastasis (BM) and skeletal-related events (SREs) in patients (pts) with stage IIIB and IV lung cancer-CSP-HOR 13. J Thorac Oncol 9: 231-238, 2014.

2. Tsuya A, Kurata T, Tamura K and Fukuoka M: Skeletal metastases in non-small cell lung cancer: A retrospective study. Lung Cancer 57: 229-232, 2007.

3. Oster G, Lamerato L, Glass AG, Richert-Boe KE, Lopez A, Chung K, Richhariya A, Dodge T, Wolff GG, Balakumaran A and Edelsberg J: Natural history of skeletal-related events in patients with breast, lung, or prostate cancer and metastases to bone: A 15-year study in two large US health systems. Support Care Cancer 21: 3279-3286, 2013.

4. Yoneda T and Hiraga T: Crosstalk between cancer cells and bone microenvironment in bone metastasis. Biochem Biophys Res Commun 328: 679-687, 2005.

5. Saylor PJ and Smith MR: Bisphosphonates and other bone-targeted therapies. Cancer Chemotherapy and Biotherapy: Principles and Practice, 5th Ed, Chabner BA, Longo DL (eds), Wolters Kluwer Lippincott Williams \& Wilkins, Philadelphia pp 732-745, 2011.

6. Roelofs AJ, Thompson K, Gordon S and Rogers MJ: Molecular mechanisms of action of bisphosphonates: Current status. Clin Cancer Res 12: 6222s-6230s, 2006.

7. Rosen LS, Gordon D, Tchekmedyian S, Yanagihara R, Hirsh V, Krzakowski M, Pawlicki M, de Souza P, Zheng M, Urbanowitz G, et al: Zoledronic acid versus placebo in the treatment of skeletal metastases in patients with lung cancer and other solid tumors: A phase III, double-blind, randomized trial-the Zoledronic acid lung cancer and other solid tumors study group. J Clin Oncol 21: 3150-3157, 2003.

8. Rosen LS, Gordon D, Tchekmedyian NS, Yanagihara R, Hirsh V, Krzakowski M, Pawlicki M, De Souza P, Zheng M, Urbanowitz G, et al: Long-term efficacy and safety of zoledronic acid in the treatment of skeletal metastases in patients with nonsmall cell lung carcinoma and other solid tumors: A randomized, Phase III, double-blind, placebo-controlled trial. Cancer 100: 2613-2621, 2004.

9. Hirsh V, Major PP, Lipton A, Cook RJ, Langer CJ, Smith MR, Brown JE and Coleman RE: Zoledronic acid and survival in patients with metastatic bone disease from lung cancer and elevated markers of osteoclast activity. J Thorac Oncol 3: 228-236, 2008.

10. Hirsh V, Tchekmedyian NS, Rosen LS, Zheng M and Hei YJ: Clinical benefit of zoledronic acid in patients with lung cancer and other solid tumors: Analysis based on history of skeletal complications. Clin Lung Cancer 6: 170-174, 2004.

11. Bamias A, Kastritis E, Bamia C, Moulopoulos LA, Melakopoulos I, Bozas G, Koutsoukou V, Gika D, Anagnostopoulos A, Papadimitriou C, et al: Osteonecrosis of the jaw in cancer after treatment with bisphosphonates: Incidence and risk factors. J Clin Oncol 23: 8580-8587, 2005.

12. Khosla S, Burr D, Cauley J, Dempster DW, Ebeling PR, Felsenberg D, Gagel RF, Gilsanz V, Guise T, Koka S, et al. Bisphosphonate-associated osteonecrosis of the jaw: Report of a task force of the American Society for Bone and Mineral Research. J Bone Miner Res 22: 1479-1491, 2007.

13. Vahtsevanos K, Kyrgidis A, Verrou E, Katodritou E, Triaridis S, Andreadis CG, Boukovinas I, Koloutsos GE, Teleioudis Z, Kitikidou K, et al: Longitudinal cohort study of risk factors in cancer patients of bisphosphonate-related osteonecrosis of the jaw. J Clin Oncol 27: 5356-5362, 2009.

14. Van Poznak CH, Temin S, Yee GC, Janjan NA, Barlow WE, Biermann JS, Bosserman LD, Geoghegan C, Hillner BE, Theriault RL, et al: American Society of Clinical Oncology executive summary of the clinical practice guideline update on the role of bone-modifying agents in metastatic breast cancer. J Clin Oncol 29: 1221-1227, 2011. 